\title{
浅析汉印语音对比和教学建议
}

\section{COMPARE ANALYSIS BETWEEN CHINESE AND INDONESIAN PHONETICS AND TEACHING SUGGESTION}

\author{
Lydia Anggreani \\ Chinese Department, Faculty of Humanities, BINUS University \\ Jln. Kemanggisan Ilir III No. 45, Kemanggisan - Palmerah, Jakarta 11480 \\ lydiaanggreani@gmail.com
}

摘要

随着中国和印尼友好关系的进一步发展, 再加上印尼华人数量比例较多, 对于汉语的学习更加重要。 本文通过汉语与印尼语的语音对比, 把汉语的语音规律呈现出来, 为汉语语音教学提供思路, 还对印尼 学生在学习汉语语音时提出了教学建议, 从而更好地完善对外汉语语音教学。

关键词: 汉语 印尼语 语音对比 语音教学

\begin{abstract}
With further development of friendly relations between China and Indonesia, coupled with a growing number of Indonesian Chinese, learning Chinese language is getting more important. Article compared Chinese and Indonesian voice and showed the voice of the Chinese language. Moreover, article provided ideas for Chinese phonetics teaching and for Indonesian students in learning Chinese pronunciation. In addition, article puts forward suggestions in order to improve foreign language teaching.
\end{abstract}

Keywords: Chinese language, bahasa Indonesia, Phonetics teaching 


\section{前言}

目前, 中国的对外汉语教学的发展, 可谓成绩喜人。据不完全统计, 目前全世界正在把汉语 作为第二语言进行学习的人数已经超过 3000 万, 超过 2500 所大学 (分布在 100 多个国家和地区) 在教授汉语。中国在 69 个国家和地区建有孔子学院, 总数已有 238 所。而中国政府和印尼政府 的文化交流越来越多, 友好关系进一步发展, 且印尼的华人很多。华人和本地人都有强烈学习 汉语的愿望, 汉语热在印尼有越来越好的发展趋势。进行汉语、印尼语的语音对比研究对于印 尼的对外汉语教学和两国的文化交流都有非常重大的意义。因此, 作为一名对外汉语专业讲师 来说, 扩大汉语印尼语语音的对比研究范围, 不断补充完善对两者的研究资料, 对于将来其他 对外汉语教师希望有很大的帮助。本文通过课堂观察来进行研究, 课堂观察就是所说的 “听课”, 课堂观察作为教师学习的一种重要形式。本文的研究对象是建国大学中文系一年级（甲班）非 汉语基础的学生, 所用的教是《看图说话》一年级。经过一学期的课程, 本人试着写出一篇关 于对外汉语语音对比和教学建议, 希望将来有助于其他对外汉语教师在研究非汉语基础的印尼 学生。

说到语音, 首先必须了解语音的物理属性, 具有音高、音强、音长、音色四个要素。换言 之, 语音是人类说话的声音, 是具有意义内容的语言成分的外部形式。评判一种语言是否学得 好不好的首要标准是, 是否正确掌握语音, 吐字、行腔、轻重快慢、升降变化掌握到位。因此, 汉语、印尼语的语音差异可体现在以下方面:

\section{汉语、印尼语的声调差异}

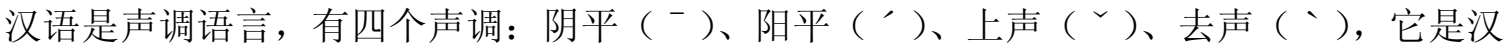
语音节的一部分, 关系到词的具体含义, 而印尼语没有声调, 是非声调语言。在印尼语里, 声 音的高低没有区别意义的作用, 只表示对话语里某一部分的强调, 如 ayo, 不管念成什么音高形 式, 它都只是感叹词, 只有一个意义, 表示邀请或命令: 来, 来吧。这就与汉语不同了, 在汉 语里, 声调是整个音节的音高变化形式, 即声调具有区别意义的作用, 如, 同一个音节 “ [PA]” 至少可以念成四种音高形式， [PA55]（巴）、[PA35（拔)、[PA214]（把）、[PA51（爸)。

\section{汉语、印尼语元音音素的差异}

汉语根据不同的口形和舌位, 可分为舌面元音 (7 个)、舌尖元音 (2 个)、卷舌元音 (1 个), 它们分别是:

舌面元音:

$\mathrm{a}[\mathrm{A}]$ 舌面、央、低、不圆唇元音

o [o] 舌面、后、半高、圆唇元音

$\mathrm{e}[\mathrm{y}]$ 舌面、后、半高、不圆唇元音

$\hat{e}[\varepsilon]$ 舌面、前、半低、不圆唇元音

$i[i]$ 舌面、前、高、不圆唇元音

$\mathrm{u}[\mathrm{u}]$ 舌面、后、高、圆唇元音

ü[y] 舌面、前、高、圆唇元音 
舌尖元音:

$-i[1]$ 舌尖前、高、不圆唇元音

$-i[\imath]$ 舌尖后、高、不圆唇元音

卷舌元音:

er [ə] 卷舌、央、中、不圆唇元音

除此之外, 汉语还有ai、ei、ao、ou、ia、ie、ua、uo、üe、iao、iou、uai、uei 等 13 个复合元音。

印尼语根据舌位的高低、前后具体分为高元音 ( 2 个)、中元音 (3 个)、低元音 (1 个); 前 元音 (2 个)、央元音 (2 个)、后元音 (2 个), 共有 9 个元音音素, 包括 6 个单元音和 3 个复 合元音, 他们分别是

单元音:

$\mathrm{a}[\mathrm{a}]$ 中、央、低唇元音

$\mathrm{e}[\varepsilon][\mathrm{e}]$ 前、低、平唇元音 lebar; [ ə ] 中、央、平唇元音； lemah

i $[\mathrm{i}$ ] 前、高、平唇元音

o [ o ] 后、低、圆唇元音

$\mathrm{u}[\mathrm{u}$ ] 后、高、圆唇元音

同样，印尼语里也有复合元音，但跟汉语相比少了许多，只有 3 个: ai、au、oi。 经过比较可以得出以下结论:

1、印尼语里 $\mathrm{o}$ 的发音与汉语略有不同, 汉语中是舌面后半高圆唇元音, 印尼语中是后低圆唇元 音, 也就是说印尼语里的 o 比汉语汉语拼音字母的 o 要开得更大些。

2、印尼语里没有u这个元音, 它的发音状况与 $\mathrm{i}$ 基本相同, 但唇形拢圆, 因此对印尼学生来说 学好花的发音有点困难。

3、印尼语里没有卷舌元音 er, 对于单纯受印尼语影响的学生来说, 碰到卷舌元音 er 与舌尖后 音 zh、ch、sh 组合时，可能不容易发好这个音，容易发成舌尖前音。

4、印尼语里没有舌尖前 $-i$ 、舌尖后 $-i$, 因此也没有汉语所说的整体认读音节, 但这个有其规定 搭配的声母 z、c、s 和 zh、ch、sh。印尼学生在发 zh 组音时, 很容易把它们跟 $z 、 c 、 s$ 和 j、q、x 混在一起。

5、印尼语里没有送气不送气的声母, 很多初学汉语的印尼学生, 往往会将送气与不送气声母混 淆起来。如把 “肚子饱了” 说成 “兔子跑了” 等等。有的学生即使有意识地发送气音, 送气 也往往不足。

\section{汉语、印尼语辅音音素的差异}

汉语辅音:

汉语共有辅音声母 21 个, 从发音部位上看, 可以分为七类, 分别是: 双唇音 ( $b 、 p 、 m)$ 、 唇齿音 $(f)$ 、舌尖前音 $(z 、 c 、 s) 、$ 舌尖中音 $(d 、 t 、 n 、 1)$ 、舌尖后音 $(z h 、 c h 、 s h 、 r)$ 、舌 面前音 $(j 、 q 、 x)$ 、舌面后音 $(g 、 k 、 h)$; 从发音方法上看, 可以分为五类, 分别是: 塞音 ( $b$ 、

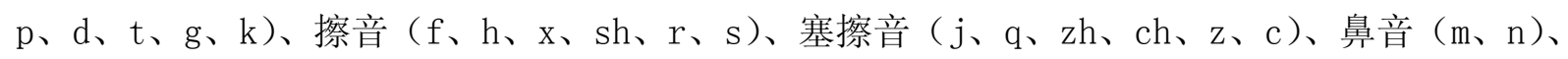
边音 (1); 按气流的强弱又可分为送气音与非送气音。 


\section{印尼语辅音:}

印尼语共有辅音音素 25 个, 从发音部位上看, 也可分为七类, 分别是: 双唇音 $(\mathrm{p} 、 \mathrm{~b} 、 \mathrm{~m}$ 、

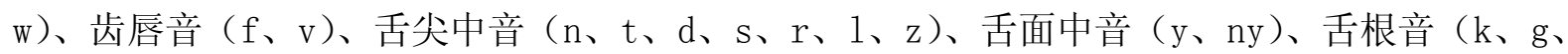
$k h 、 n g 、 q)$ 、混合舌叶音 $(s y 、 c 、 j)$ 、喉音 $(h)$ 。从发音方法上看, 同样也分为七种, 分别是:

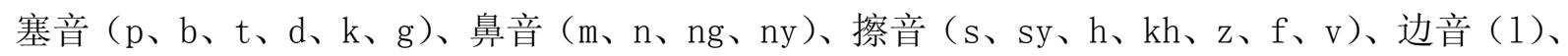
塞擦音 $(c 、 j)$ 、半元音 $(y, w)$ 、颤音 $(r)$ 。如以上，印尼语里没有送气与非送气之分。

经过对比后可得出汉语印尼语辅音差异如下:

1、印尼语辅音音素比汉语丰富, 没有舌尖后音 $\mathrm{zh}$ 、 ch、sh, 但有半元音 w、y, 此外, 擦音多 了 $\mathrm{kh} 、 \mathrm{sy} 、 \mathrm{v}$ ，鼻音多了 $\mathrm{ng} 、 \mathrm{ny}$ 。

2、汉语、印尼语都有音素 $r$, 但发音方法却大有不同。汉语里 $r$ 是舌尖后、擦音, 发音时舌尖 上尧接近硬腭前部, 形成窄缝, 软腭上升, 堵塞鼻腔通路, 声带颤动, 气流带音; 而在印尼 语里 $r$ 是颤音, 发音时舌尖抬向齿龈后部, 当气流冲出时, 舌尖上下颤动, 舌尖要放松, 不 能紧张, 不能太大力。这是跟汉语语音最大的一个不同点之一, 对于单纯受印尼语影响的学 生来说, 要注意对其讲清两者的不同, 解释清楚正确的发音方法和规律, 否则可能会容易发 成印尼语的颤音。

3、印尼语里的混合舌叶音 $j 、 c$, 发音部位是舌尖舌面与前腭接触, 这与汉语有所不同, 可能 会有点难度, 特别是 $\mathrm{c}$ 。在汉语里, $\mathrm{c}$ 发音时, 舌尖轻轻抵住齿背, 软腭上升, 堵塞鼻腔通 路, 同时要送气。由于舌尖与舌叶这两个发音部位比较难把握, 而且印尼语没有送气音, 因 此, 对印尼学生来说这个音是有难度的。

4、汉语里的舌面前音 $\mathrm{q} 、 \mathrm{x}$, 在印尼语里只出现在借词中, $\mathrm{q}$ 念 $\mathrm{k}$ 音, 只作起音, 不作音尾, 如 quota, 读成kuota。而 $\mathrm{x}$ 做起音时念成 $\mathrm{s}$, 只出现于借词中的第一个音节, 如 xantat、xilofon; 做尾音时念成 $k s$, 写成 $k s$, 如 latéks。如果 $\mathrm{x}$ 后面紧接的是辅音, ks 自成一个音节, 如ékspor; 如果后面紧接的是元音, 那么 $\mathrm{ks}$ 中的 $\mathrm{s}$ 成为后面元音的起音, 如 taksi。 $\mathrm{x}$ 在作起音时与汉 语的辅音声母相似, 读作 $\mathrm{s}$, 因此很多印尼学生可能会直接把 $\mathrm{x}$ 读成 $\mathrm{s}$ 。

\section{汉语、印尼语音节结构差异}

汉语的音节是由 21 个声母和 39 个韵母按照一定的规律拼合后得到的, 共有如下 10 中组合 方式 (其中, $\mathrm{V}=$ 元音, $\mathrm{K}=$ 辅音):

1、V 如: a (啊)、 $\mathrm{O}$ (哦)、e（饿）

2、VV 如: ai (唉)、ao (奥)、ou (呕)

3、VK 如: an (安)、en (恩)、(y) in (因)

4、VVV 如: iou (优)、uei (威)、uai (歪)

5、VVK 如: ian (烟)、uan（弯）、uen（问）

6、KV 如: ba (爸)、ce (册)、na (那)

7、KVV 如: bai (白)、cui (崔)、pei (陪)

8、KVK 如: can (餐)、ben (奔)、qun (群)

9、KVVK 如: huan (还)、jian（间）、1uan（乱）

10、KVVV 如: qiao (桥)、kuai (快)、shuai（帅）

印尼语的音节组合有 11 种方式，分别如下：

1、V 如：i-bu（母亲）、a-nak（孩子)、u-mur（年龄）

2、VK 如: an-da (你)、un-di (抽奖)、 im-1ek (春节) 
3、 KV 如: bi-bi (姑母)、ca-ri (找)、 da-du（骰子）

4、KVK 如: ban-da (财富)、cen-til (风流)、bon-sai (盆景)

5、KKV 如: gra-dis (免费)、kre-dit（信用卡)、pre-man（流讯）

6、KKVK 如: prak-tis (实用)、trak-tir (请客)、spon-tan（自发的）

7、VKK 如: ang-ka (数字)、eng-gan (不顾)、ong-kos（费用）

8、KVKK 如: mung-kin (也许)、ping-gir (逃避)、rong-ga（洞）

9、KKVKK 如: trans-fer (转发)、kom-pleks (复杂)、blong ( )

10、KKKV 如: stra-ta (层次)、stra-te-gi (战略)

11、KKKVK 如: stres（压力）、in-struk-si (指示)、struk-tur（结构）

经比较得出结论如下:

1、印尼语里没有 VV 组合规律, 虽然印尼语是双元音, 但在印尼语里不能再划分为 VV, 而且 不能独立成词, 但汉语的双元音可以独立成词。

2、印尼语的单词一般都比汉语长, 它的 “双音节” 或 “多音节” 可能在汉语里只是个 “单音 节” 或者 “双音节”。印尼语的基本词一般是由 1 至 5 个音节组成的, 而汉语一般是一个音 节就是一个汉字的读音。

3、印尼语的辅音位置比较自由, 可以在音节开头、中间或是末尾, 但汉语里的辅音大都在音 节开头或末尾, 在末尾出现的辅音只限于 $\mathrm{n}$ 和 $\mathrm{ng}$, 且汉语没有两个辅音相连的音节, $\mathrm{zh}$ 声 母、ng 韵尾都是双字母的音素符号, 表示一个辅音音位。

4、汉语里的三个鼻辅音 $m 、 n 、 n g$ 可以独立成为一个音节, 表示叹词 “呣”、 “嗯” 等, 而印尼 语里没有这种现象。

\section{印尼学生在学习汉语语音方面常见偏误}

本人在课堂上对课文进行了以下的步骤:

1. 教师采用图片对课文进行了导入在此过程中让同学们一起跟着老师读。打开书本带领同学们 逐句朗读课文并和同学们一起进行了朗读。

2. 点几个学生重读课文, 若有发音不准的话, 及时修正。

3. 讲完了一节课最后又一起朗读了课文。

4. 带领同学们对课文内容进行了回忆。

对于学生的语音偏误, 本人经过以上的课堂步骤做出了一下的总结, 本人认为印尼学生在 学习汉语语音过程当中常见的偏误如下:

(一) 发不好 $z 、 c 、 s$ 和 zh、ch、sh、r

印尼语里没有这两组声母发音相应的声母, 发音有所不同。导致学生在学习这类声 母时往往找不到感觉。有的学生将舌尖后音 $\mathrm{zh}$ 、ch、sh 读成舌叶音, 或误将舌尖后元音 $-i$ 读成单元音 $i$ (衣), 听起来成了舌面音 $j 、 q 、 x$ 。还有, 有的学生不能将就 $j 、 q 、 x$, zi、ci、si与 zhi、chi、shi 的区别发清楚。

（二）分不清送气和不送气声母的区别

印尼语中声母没有送气和不送气的, 虽然有一些声母有的是送气, 但并没有区别意 义的作用。学生在发音时, 缺乏音位对立意识, 因此在这方面就会经常出现出现偏误。 如把 “肚子饱了” 读成 “兔子跑了”。有的学生即使有意识到发送气音, 可是发出来的并 不到位。 
（三）因罗马字写法相同而产生混淆

汉语拼音是用罗马字标注的, 不少学生在看到汉语表音的罗马字时, 会发成使用相 同符号的母语的辅音声母。如汉语的 “ $\mathrm{t}$ ” 读成印尼 “ $\mathrm{d}$ ”, 前者是舌尖顶住上齿龈, 送气, 声带不颤动。后者是舌尖中音, 不送气。

(四) 对印尼学生来说, 最难发的单元音是ü。 前突出, 作出最小的圆形, 难度较大。不少印尼学生在发ü时, 或者因为圆唇不够而发成 近似 $i$ 的元音; 或因注意圆唇而使他们不自觉的将舌位后移, 发成类似 $u$ 或 $i+u$ 的音。 如 “月饼” 读成 “叶饼”。还有, 当 $u$ 与 $j 、 q 、 x$ 相拼时, 由于 $u$ 上面的两点被省去, 就与 $\mathrm{u}$ 无异，许多学生会将把它读成 $\mathrm{u}$ 而不是 $\ddot{u}$ 的音。由于印尼语 $x 、 z 、 c 、 s 、 z h 、 c h 、 s h ~$ 后都用相同的罗马字 i 标音, 学生常会把舌尖前元音 [ 1 ] 与舌尖后元音 [ $]$ 读成罗马字的 i 音。

\section{教学建议}

为了使学生更深入地了解和掌握汉语的语音。本人经过教学观察做出了对外汉语语音教学 的教学建议, 由于本人的能力有限所以只做出了几项建议。希望这项教学建议对于对外汉语界 有帮助, 尤其语音方面。

1. 教汉语拼音之处, 应该强调汉语拼音的作用是标志普通话的发音法, 它的罗马字母即使与学 生的母语使用的字母相同，但表示的音并不相同。

2. 印尼学生母语中没有的声母和韵母比较难学的。可以采用各种直观或简便的方法, 说明具体 的发音部位和发音方法, 增加学生的感性认识。如在教 zh、ch、sh、r 可以让学生把手指 放在齿间, 让学生体会翘舌的感觉。

在教送气与不送气的时候, 应特别强调它们在区别意义上的作用, 强调发好这些声母的重 要性。为了让学生找到感觉, 教师一般会用一张薄纸, 放在嘴唇前, 用力发送气音, 让学 生看自己面前的纸抖动。

3. 印尼语离没有声调的出现, 导致印尼学生在学习汉语过程中有一定的难度。本人按照课堂上 的观察有的学生在单独读单音节和双音节时, 发出的音是正确的 (情况是在教师的领读下)。 若让他们自己读的时候, 他们往往抓不住正确的声调。对于声调的教学建议, 一、要加强 单双音节或双音节以上的词语或短语的声调训练, 学生在学习语音阶段结束之后, 仍要经 常复习。还要结合常用词语和常用短语加大练习量, 让学生反复练习而熟悉并掌握正确的 汉语声调; 二、加强课文的领读、范读。如: 口语课应该加强句子的整体输入与输出, 最 好能让一个学生背诵一些常用句子, 尽量注意声调。

课堂上除了教师经常领读、范读, 是学生多接触正确的语音之外, 也可以让学生在别人朗 读时, 注意发现其发音不正确的地方。教师也要加以及时的纠正, 鼓励学生多听标准的汉 语录音与电视节目、电台播音员正确的句调、语调, 边听边模仿; 鼓励学在课下多用汉语 交流, 说错也没关系, 只要学生敢用汉语说话就好。

\section{结论}

综上所述, 汉语和印尼语在元音、辅音、声调、音节结构上的差异都比较明显的两种语言, 本文通过对比两种语言的不同之处, 总结归纳印尼学生在学习汉语时容易混淆的问题, 并对对 外汉语教师提出相应的教学建议, 为其在对外汉语教学中提供一些思路。以上是本篇论文对汉 
语印尼语语音对比的一些浅显分析, 对此, 对外汉语教师在其教学过程中应多积累素材, 多积 累教学方法, 形成一定的理论, 不断丰富对外汉教学的理论研究。

\section{参考文献}

高惠敏.（2005). 印尼语语音对汉语普通话语音学习的影响. 北京:海外华文教育.

黄伯荣、廖序东主编. (2000). 现代汉语 (增订版). 北京: 高等教育出版社.

林奉、王理嘉.（1992）.语音学教程. 北京：北京大学出版社.

朱刚琴. 2008. 基础印度尼西亚语.（1）.世界图书出版公司

何微、林齐倩. (2002) 初级班口语教学中的语音问题及对策一一对欧美学生发音调查给我们的 启示. 专辑。 